\title{
Evaluation of a Recombinant Measles Virus as the Expression Vector of Hepatitis C Virus Envelope Proteins
}

\author{
Yuri Kasama $^{1}$, Masaaki Satoh ${ }^{1}$, Makoto Saito ${ }^{1}$, Seiji Okada ${ }^{2}$, Chieko Kai ${ }^{3}$, \\ Kyoko Tsukiyama-Kohara ${ }^{*}$
}

${ }^{1}$ Department of Experimental Phylaxiology, Faculty of Life Sciences, Kumamoto University, Kumamoto, Japan; ${ }^{2}$ Division of Hematopoiesis, Center for AIDS Research, Kumamoto University, Kumamoto, Japan; ${ }^{3}$ Laboratory of Animal Research Center, Institute of Medical Science, University of Tokyo, Tokyo, Japan.

Email: *kkohara@kumamoto-u.ac.jp

Received June $16^{\text {th }}, 2011$; revised July $18^{\text {th }}, 2011$; accepted July $29^{\text {th }}, 2011$.

\begin{abstract}
Measles virus $(M V)$ is a negative strand RNA virus of the family Paramyxoviridae, and the attenuated Edmonston-B strain can be engineered by the reverse genetics system. Here we constructed the recombinant Edmonston strain of measles virus $(M V-E d)$ that expressed hepatitis $C$ virus (HCV) envelope proteins ( $r M V$-E1E2). The rMV-E1E2 successfully expressed HCV E1 and E2 proteins. To evaluate its immunogenicity, NOD/Scid/Jak3null mice that were engrafted with human peripheral blood mononuclear cells (huPBMC-NOJ) were infected with this rMV-E1E2. Although human lymphocytes could be isolated from the spleens of mock-infected mice during the 2-weeks-long experiment, the proportion of mice that were infected with $M V$ or $r M V$-E1E2 was decreased in a viral dose-dependent manner. Over $10^{3} P F U$ of virus infection decreased the human PBL to less than 5\%. Significant decrease of B cell population in human PBL from $r M V$-E1E2 infected NOD-SCID mice and decrease of T cell population in those from MV infected mice were observed. Human antibody production in these mice was also examined. Thus, the results in this study may contribute for future improvement of recombinant vaccine using measles virus vector.
\end{abstract}

Keywords: $M V, H C V$, E1, E2, Human PBMC, NOD/Scid/Jak3null Mouse

\section{Introduction}

Measles virus (MV) is classified into the family Paramyxoviridae. Its genome is composed of 15,894 nucleotides, which encodes a nucleocapside protein $(\mathrm{N})$, phospho protein $(\mathrm{P})$, matrix $(\mathrm{M})$, haemaglutinin $(\mathrm{H})$, fusion (F), and viral polymerase (L). The establishment of the reverse genetic system for the rescue of an attenuated Edmonston-B stain from cloned DNA [1] enabled to develop measles virus as virus expression vector. Subsequently, a number of foreign proteins have been efficiently expressed. Efforts to develop vaccines using recombinant MV expressing different proteins derived from dengue virus [2,3], human immunodeficiency virus [4], HPV[5], SARS [6], or West Nile virus [7] have been reported.

The MV vaccine is a well-known, live-attenuated vaccine and has proven to be one of the safest, most stable, and effective human vaccines [8]. This vaccine is produced on a large scale in many countries and used at low cost through the Extended Program on Immunisation of the WHO $[9,10]$. While this vaccine has been shown to induce life-long immunity with a single dose, boosting is effective. Thus, MV possesses a high potential for the vaccine vector.

Hepatitis $\mathrm{C}$ virus $(\mathrm{HCV})$ is a member of the Flaviviridae family and is the causative agent of both chronic hepatitis and hepatocellular carcinoma (HCC) [11]. 170 million people are infected with HCV worldwide $[12,13]$. Despite prevention efforts and advanced treatment strategies, including combined PEGylated IFN- $\alpha$ and ribavirin therapy $[14,15]$, the clinical efficacy of this treatment is limited $[16,17]$. Alternative novel antiviral agents that have been shown to elicit effective responses in chronically infected patients, such as inhibitors of viral protease, helicase, and polymerase, are currently being developed but are expensive [18]. Therefore, the development of an effective vaccine is important.

Immunological approaches to control HCV infection 
have proven to be ineffective, in part because $\mathrm{HCV}$ adapts to escape from the host immune system [19]. Furthermore, a high percentage of immunocompetent individuals are infected by HCV despite their ability to mount an active immune response [20]. A preventive $\mathrm{HCV}$ vaccine is required to protect unexposed individuals from $\mathrm{HCV}$ infection. This vaccine will most likely need to target the viral envelope glycoproteins, E1 and E2.

HCV research has long been hampered by the lack of an animal model that reproduces HCV infection in humans. The model in which severe combined immunodeficient (SCID) mice are transplanted with human peripheral blood mononuclear cells (PBMC) is a well-established system to study human immunity (hu-PBMC-SCID). This mouse develops all human lymphoid cell lineages that repopulate the animal's lymphoid organs. Our group previously generated the non-obese diabetic (NOD)/SCID/ Janus kinase 3 (Jak3) knockout (NOJ) mouse model and then established a human hemolymphoid system in this mouse $[21,22]$. In this study, we infect human PBMCtransplanted NOJ mice with MV and rMV-E1E2 and then characterise their infectivity and effects in the transplanted human cells.

\section{Materials and Method}

\subsection{Cells}

B95a cells, a marmoset B cell line[23], were used for viral titration and rescue, and were maintained in RPMI 1640 medium supplemented with $10 \%$ heat-inactivated foetal calf serum (FCS).

\subsection{Plasmid Construction and Viral Rescue}

The cDNAs encoding HCV E1 and E2 were obtained from the plasmid HCR6CNS2 [24]. We used replication-competent MV-based vectors (pMV; MV Edmonston strain) [1]. The E1 and E2 cDNAs were cloned into the Fse I site of pMV and the resulting clone, pMV-E1E2, was used to rescue the infectious recombinant MV expressing the HCV envelope glycoproteins (rMV-E1E2), as reported previously $[25,26]$.

\subsection{Generation of Humanised Mice}

Mice were reconstituted as described previously $[21,22]$. The NOD/SCID/JAK $3{ }^{\text {null }}$ strain was established by backcrossing JAK3 $3^{\text {null }}$ and the NOD Cg-Prkdc ${ }^{\text {Scid }}$ strains for ten generations. All animal experiments were performed according to the guidelines of Institutional Animal Committee or Ethics Committee of Kumamoto University.

\subsection{Preparation of Human Blood Leukocytes and Transplantation}

Peripheral blood mononuclear cells were isolated from blood donors using Ficoll-Hypaque density gradient centrifugation. A total of $5 \times 10^{6}$ cells were transplanted into the spleen of irradiated (2 Gy) 4-weeks-old mice.

\subsection{MV and rMV-E1E2 Infection}

We injected 100 - 104 PFU of MV or $100-102$ or 104 PFU of rMV-E1E2 intraperitoneally for MV and rMV-E1E2 infection, respectively. As a negative control, a group of mice was injected with RPMI 1640. Mice were moni- tored for 2 weeks and then euthanised. The spleens and peripheral blood were collected for analysis.

\subsection{Flow Cytometry}

Isolated splenocytes were stained with APC-Cy7-conjugated anti-mouse CD45 (BD Pharmin-gen) to detect the murine lymphocytes and either APC - or pacific blueconjugated anti-human CD45 (DAKO), hCD3-PE/Cy7, hCD19-FITC, hCD4-PE, and hCD8-PB (BD Biosciences) to detect human lymphocytes. All data were analysed using FlowJo (Tree Star).

\subsection{Confirmation of Viral Infection}

The viral infection of the human lymphocytes was con-firmed using co-culture with B95a cells followed by RT-PCR. Suspensions of isolated splenocytes were co-cultured with B95a cells and the formation of cytopathic effects (CPEs) was monitored for 2 weeks. Additionally, RNA was isolated from the supernatant of the co-cultures using ISOGEN-LS (Nippon gene) according to manufacturer's instructions. MV RNA was detected using RT -PCR with the sense primer, 5'-ACTCGGTATCACTGCCGAGGATGCAAGGC-3' (1256 - 1284) and antisense primer 5'-CAGCGTCGTCATCGCTCTCTCC-3'(2077 2056) or 5'-ATGGCAGAAGAGCAGGCACG-3'(1807 1826). HCV E1 or E2 was amplified using E1-S-1051 5'-CCGTTGCTGGGTGGCACTTA-3 and E1-AS-1314 5'-ATCATCATGTCCCAAGCCAT-3' or E2-S-1600 5'CTGGCACATCAACAGGACTG-3' and E2-AS-1960 5'AAGGAGCAGCACGTCTGTCT-3'.

\subsection{ELISA}

Anti-MV antibody titers were determined by using an ELISA assay. 96-well plates were coated with a $25 \mathrm{~g} / \mathrm{ml}$ solution of MV-infected B95a lysate or recombinant E2-expressing baculovirus-infected Sf9 lysate as antigen, respectively. The plates were consecutively incubated with sera $(1: 100)$ recovered from hu-PBL-NOJ mice, peroxidase-conjugated rabbit-human IgG (DAKO), and TMB Peroxidase EIA Substrate Kit (Bio-Rad) at $37^{\circ} \mathrm{C}$ for $1 \mathrm{~h}$. Optical density values were measured at $450 \mathrm{~nm}$.

\subsection{Western Blot Analysis}

Total protein extracts from E2-expressing baculovirus-in- 
fected Sf9 lysate were separated by SDS-PAGE. The primary antibodies used for western blots were as follows: sera from mice $(1: 100)$ and anti-E2 monoclonal antibody (1:5000). Peroxidase-conjugated secondary antibodies were added and incubated with the mixture for $1 \mathrm{~h}$ at room temperature. Bound antibody was visualized using ECL reagent (Amersham Bioscience) with LAS1000 scanner (Fuji film).

\section{Results}

\subsection{Construction of Recombinant Measles Virus Expressing HCV E1 and E2 Proteins}

The HCV genes corresponding to the envelope proteins $\mathrm{E} 1$ and $\mathrm{E} 2$ were sub-cloned in between the $\mathrm{N}$ and $\mathrm{P}$ genes of the MV vector (Figure 1(a)). The HCV E1 and E2 genes included the putative signal peptide sequences at the $\mathrm{N}$ terminus and the transmembrane domain at the $\mathrm{C}$ terminus [27]. The plasmid vector pMV-E1E2 was introduced with supporting plasmids into 293T cells to rescue the recombinant viruses. The expression of the HCV E1 and E2 proteins by rMV-E1E2 was examined by western blotting (Figure 1(b)).

\subsection{Infection of hu-PBMC-NOJ Mice with MV and rMV-E1E2}

All hu-PBMC-NOJ mouse infections were 14 days long. Infections with MV and rMV-E1E2 were confirmed by first co-culturing the human lymphocytes isolated from the spleens of infected mice with B95a cells and then verifying the presence of virus by RT-PCR. In all the MV $\left(10^{3}-10^{4} \mathrm{PFU}\right)$ or rMV-E1E2 $\left(10^{4} \mathrm{PFU}\right)$-infected hu-PBMC-NOJ mice, CPEs were observed in co-cultures containing splenocytes that were isolated from infected mice (Table 1). The results of the co-culture assays are in agreement with results that were obtained by RT-PCR; positive bands were observed in the mice infected with $10^{3}-10^{4} \mathrm{PFU}$ of MV and $10^{2}, 10^{4} \mathrm{PFU}$ of rMV-E1E2 (Table 1). These results demonstrate that the rescued MV and rMV-E1E2 are able to infect transplanted human PBMCs.

\subsection{Proportion of Engrafted Human Lymphocytes in MV- and rMV-E1E2-Infected Hu-PBMC-NOJ Mice}

The splenocytes that were isolated from infected mice were analysed using flow cytometry to determine the proportion of human cells in the spleen (Table 1, Figure 2). In the MV-infected hu-PBMC-NOJ mice, a population of human lymphocytes was observed in the mice that were infected with $10^{0}-10^{2} \mathrm{PFU}$, whereas few human lymphocytes were observed in mice infected with $10^{3}$ $-10^{5}$ PFU. In the rMV-E1E2-infected mice, a population
rMV Ed. full genome $(15,894$ ntds.) Plasmid (pMV)
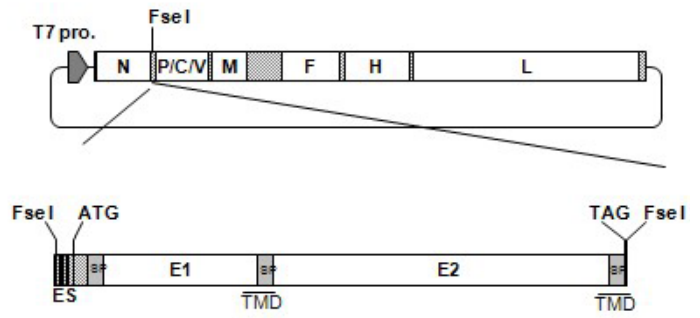

HCVE1E2 (1,776 ntds.)

(a)
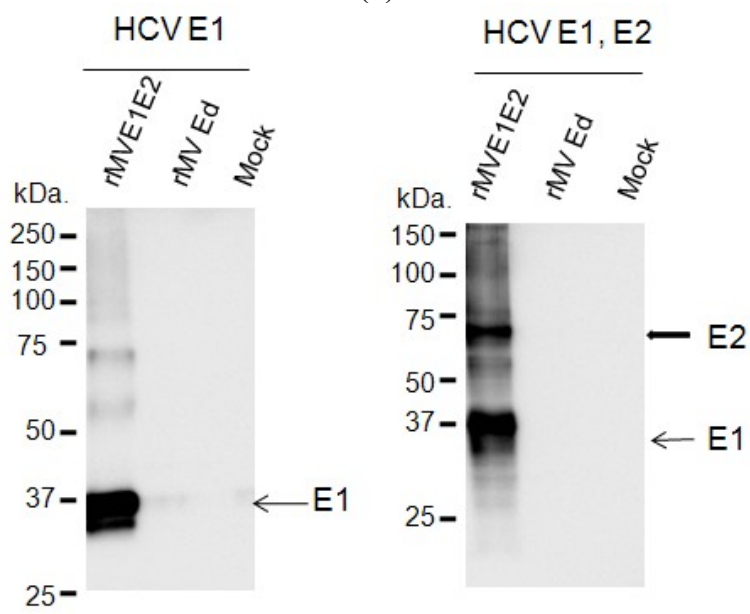

(b)

Figure 1. Construction of the recombinant MV vectors. (a) The rMV full genome vector derived from the MV-Ed strain is illustrated in the upper panel and is labelled with letters as follows: $N$, nucleocapsid; $P$, phosphoprotein; $M$, matrix; F, fusion; $H$, hemagglutinin; and $L$, large. $T 7$ indicates the T7 RNA polymerase promoter. The cDNA encoding the HCV envelope glycoproteins (E1 and E2) containing the signal peptide sequence (SP) and the transmembrane domain (TMD, underlined) regions, the $\mathrm{N}$ gene end signal (E), the $\mathbf{P}$ gene start signal (S), and the intercistronic region of the $H$ protein genes at the 5 ' end, which was flanked by Fse I sites at both ends, was introduced into the unique Fse $I$ site in between the $N$ and $P$ genes in the pMV vector. The resulting plasmid was designated pMV-E1E2. (b) The HCV E1 and E2 proteins were detected in rMV-E1E2-, rMV-Edand mock-infected B95a cells by western blot with MoAb 384 for E1 and MoAb 384 and 544 for E1 and E2 (arrows).

of human lymphocytes was detected in mice that were inoculated with $10^{0}-10^{2} \mathrm{PFU}$. The ratio of human lymphocyte settlement in both groups of mice was inversely correlated with the results from the RT-PCR and coculture assays (Table 1). The B cell population of rMVE1E2 infected human lymphocytes was significantly decreased than medium control (Figure 2). In contrast, the 
Table 1. Summary of MV infection to human PBL in NOD/Scid mice.

\begin{tabular}{|c|c|c|c|c|c|c|c|}
\hline \multirow{2}{*}{ Virus } & \multirow{2}{*}{$\begin{array}{l}\text { Amount } \\
\text { of virus }\end{array}$} & \multirow{2}{*}{$\begin{array}{l}\text { No. } \\
\text { tested }\end{array}$} & \multirow{2}{*}{$\begin{array}{l}\text { huPBL settlement } \\
\left(\text { Av. }+/-\mathrm{SD}^{\circ}\right)\end{array}$} & \multirow{2}{*}{$\mathrm{CPE}^{*}$} & Viral & \multicolumn{2}{|c|}{ ELISA $^{(a)}$} \\
\hline & & & & & RNA** & MV & E2 \\
\hline \multirow[t]{3}{*}{ Mock } & 0 & 6 & $90.9 \pm 13.1$ & $0 / 6$ & $0 / 6$ & $\mathrm{ND}^{\#}$ & ND \\
\hline & $10^{0}$ & 3 & $92.7 \pm 11.2$ & $0 / 3$ & $0 / 3$ & $1 / 3$ & ND \\
\hline & $10^{1}$ & 3 & $58.4 \pm 50.6$ & $0 / 3$ & $0 / 3$ & $2 / 3$ & ND \\
\hline \multirow{5}{*}{ MV } & $10^{2}$ & 3 & $55.1 \pm 49.9$ & $0 / 3$ & $0 / 3$ & ND & ND \\
\hline & $10^{3}$ & 2 & $4.9 \pm 6$ & $2 / 2$ & $2 / 2$ & ND & ND \\
\hline & $10^{4}$ & 2 & 1.7 & $2 / 2$ & $2 / 2$ & ND & ND \\
\hline & $10^{5}$ & 2 & 3.7 & $\mathrm{NT}^{+}$ & NT & ND & ND \\
\hline & $10^{\circ}$ & 2 & 79.6 & $0 / 2$ & $0 / 2$ & ND & ND \\
\hline \multirow{3}{*}{ MV-E1E2 } & $10^{1}$ & 2 & 96.0 & $0 / 2$ & $0 / 2$ & $2 / 2$ & ND \\
\hline & $10^{2}$ & 3 & $56.2 \pm 36.2$ & $0 / 3$ & $1 / 3$ & $1 / 3$ & $1 / 3$ \\
\hline & $10^{4}$ & 4 & $0.34 \pm 0.004$ & $4 / 4$ & $4 / 4$ & ND & ND \\
\hline
\end{tabular}

${ }^{*} \mathrm{CPE}$ formation in B95a cells co-cultured with PBL from each mice; **Detection of MV or HCV-RNA in PBL by RT-PCR. +NT; not tested; ${ }^{*}$ Not detected. ${ }^{\circledR}$ Human antibody was detected by ELISA.
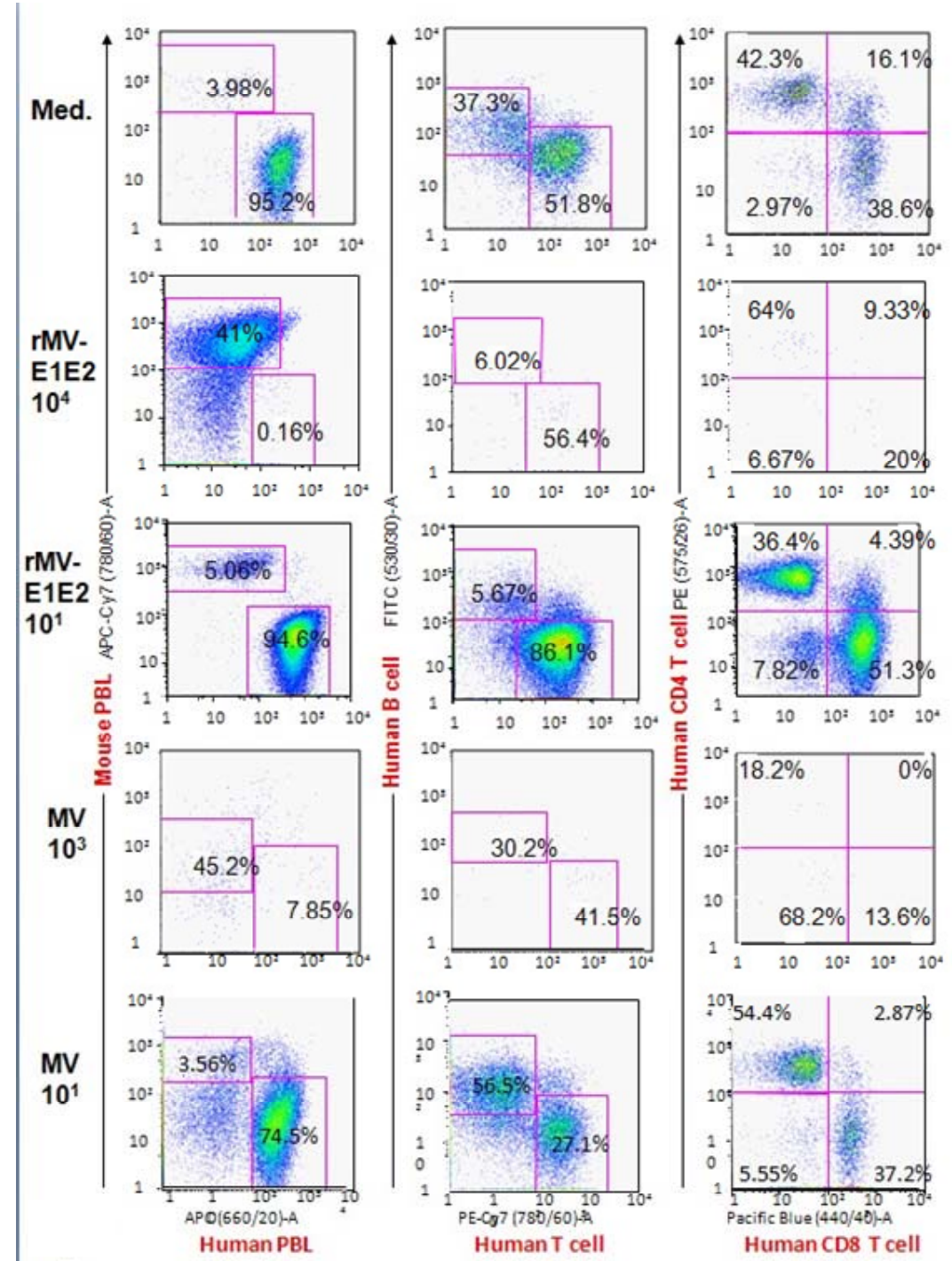

Figure 2. Flow cytometric analysis of splenocytes isolated from hu-PBMC-NOJ mice inoculated with medium, rMV-E1E2 $\left(10^{1}, 10^{4} \mathrm{PFU}\right)$, or MV-Ed $\left(10^{1}, 10^{3} \mathrm{PFU}\right)$. Splenocytes, consisting of both human and murine cells, were stained with antibodies against human or mouse CD45. Human PBL was further stained with hCD3-PE/Cy7, hCD19-FITC, hCD4-PE, and hCD8-PB. Representative flow cytometric profiles of each group of infected mice are shown. The percentage of lymphocytes are shown by numbers. 
T cell population of MV infected human lymphocytes were significantly decreased than medium control.

To examine the immune response against MV and rMV-E1E2 by the transplanted human PBMCs, we measured human MV- or HCV-specific antibodies using an ELISA with an MV-infected B95a cell lysate or recombinant HCV E2 protein. A significant amount of human antibody against MV antigens was detected in the sera from mice that were infected with MV $\left(10^{0} \mathrm{PFU} 1 / 3,10^{1}\right.$ PFU 2/3) or rMV-E1E2 $\left(10^{1} 2 / 2,10^{2}\right.$ PFU $\left.1 / 3\right)$. However, only one mouse, which was infected with $10^{2} \mathrm{PFU}$ of rMV-E1E2, generated human antibodies against HCV E2 $\left(10^{2} \mathrm{PFU} 1 / 3\right)$, which was confirmed by western blot analysis (Table 1).

\section{Discussion}

The results in this study revealed that more than $10^{3} \mathrm{PFU}$ of MV or $10^{4} \mathrm{PFU}$ of rMV-E1E2 infection decreased the human PBL to less than 5\%. We detected a significant amount of MV-specific antibodies in the rMV-E1E2-infected mice $(n=3)$. However, only one mouse produced E2-specific antibodies and no mice produced E1-specific antibodies. These results may be consistent with the hypothesis that the immunogenicity of the E1 and E2 proteins might be lower than the immunogenicity of the MV proteins, an observation that is consistent with previous studies [28-31]. Future studies are required to clarify the immune response to $\mathrm{HCV}$ envelope proteins and protection of viral infection.

In summary, we infected human PBMC-transplanted NOJ mice with MV and rMV-E1E2 and then characterised the humoral immune responses elicited by the transplanted human cells. The results in this study revealed the possibility and improvement of rMV-E1E2 and the potential and the limit of hu-PBMC-NOJ mice for the evaluation of the immunogenicity of viral proteins.

Further development of the rMV-E1E2 and hu-PBMC NOJ mouse model system will allow us to develop potential vaccine targets, which are indispensable for the development of an effective vaccine.

\section{Acknowledgements}

We would like to thank M. A. Billeter and K. Takeuchi for providing the MV Edmonston B strain rescue system, S. Iwanaga, K. Tanaka, F. Ikeda and M. Yoneda for their technical support, and M. Kohara for providing the anti-E1 and E2 antibodies. This work was supported by grants from the Ministry of Health and Welfare of Japan, and the Ministry of Education, Culture, Sports, Science and Technology of Japan.

\section{REFERENCES}

[1] F. Radecke, P. Spielhofer, H. Schneider, et al., "Rescue of Measles Viruses from Cloned DNA," EMBO Journal, Vol. 14, No. 23, 1995, pp. 5773-5784.

[2] S. Brandler, M. Lucas-Hourani, A. Moris, et al., "Pediatric Measles Vaccine Expressing a Dengue Antigen Induces Durable Serotype-Specific Neutralizing Antibodies to Dengue Virus," PLoS Neglected Tropical Diseases, Vol. 1, No. 3, 2007, pp. e96-e108. doi:10.1371/journal.pntd.0000096

[3] S. Brandler and F. Tangy, "Recombinant Vector Derived from Live Attenuated Measles Virus: Potential for Flavivirus Vaccines," Comparative Immunology, Microbiology and Infectious Diseases, Vol. 31, No. 2-3, 2008, pp. 271-291. doi:10.1016/j.cimid.2007.07.012

[4] C. Lorin, L. Mollet, F. Delebecque, et al., "A Single Injection of Recombinant Measles Virus Vaccines Expressing Human Immunodeficiency Virus (HIV) Type 1 Clade B Envelope Glycoproteins Induces Neutralizing Antibodies and Cellular Immune Responses to HIV," Journal of Virology, Vol. 78, No. 1, 2004, pp. 146-157. doi:10.1128/JVI.78.1.146-157.2004

[5] G. Cantarella, M. Liniger, A. Zuniga, et al., "Recombinant Measles Virus-HPV Vaccine Candidates for Prevention of Cervical Carcinoma," Vaccine, Vol. 27, 2009, pp. 3385-3390. doi:10.1016/j.vaccine.2009.01.061

[6] M. Liniger, A. Zuniga, A. Tamin, et al., "Induction of Neutralising Antibodies and Cellular Immune Responses against SARS Coronavirus by Recombinant Measles Viruses," Vaccine, Vol. 26, No. 17, 2008, pp. 2164-2174.

[7] P. Despres, C. Combredet, M. P. Frenkiel, et al., "Live Measles Vaccine Expressing the Secreted Form of the West Nile Virus Envelope Glycoprotein Protects against West Nile Virus Encephalitis," Journal of Infectious Diseases, Vol. 191, No. 2, 2005, pp. 207-214. doi:10.1086/426824

[8] C. Combredet, V. Labrousse, L. Mollet, et al., "A Molecularly Cloned Schwarz Strain of Measles Virus Vaccine Induces Strong Immune Responses in Macaques and Transgenic Mice," Journal of Virology, Vol. 77, No. 21, 2003, pp. 11546-11554.

doi:10.1128/JVI.77.21.11546-11554.2003

[9] D. Naniche, M. Garenne, C. Rae, et al., "Decrease in Measles Virus-Specific CD4 T Cell Memory in Vaccinated Subjects," Journal of Infectious Diseases, Vol. 190, 2004, pp. 1387-1395. doi:10.1086/424571

[10] I. G. Ovsyannikova, N. Dhiman, R. M. Jacobson, R. A. Vierkant and G. A. Poland, "Frequency of Measles Virus-Specific CD4+ and CD8+ T Cells in Subjects Seronegative or Highly Seropositive for Measles Vaccine," Clinical and Diagnostic Laboratory Immunology, Vol. 10, No. 3, 2003, pp. 411-416.

[11] A. M. Di Bisceglie, R. L. Carithers Jr. and G. J. Gores, "Hepatocellular Carcinoma," Hepatology, Vol. 28, No. 5, 1998, pp. 1161-1165. doi:10.1002/hep.510280436

[12] Global Surveillance and Control of Hepatitis C, "Report of a WHO Consultation Organized in Collaboration with the Viral Hepatitis Prevention Board, Antwerp," Journal of Viral Hepatitis, Vol. 6, No. 1, 1999, pp. 35-47. 
doi:10.1046/j.1365-2893.1999.6120139.x

[13] F. Zoulim, M. Chevallier, M. Maynard and C. Trepo, "Clinical Consequences of Hepatitis C Virus Infection," Reviews in Medical Virology, Vol. 13, No. 1, 2003, pp. 57-68. doi:10.1002/rmv.371

[14] A. Bruchfeld, L. Stahle, J. Andersson and R. Schvarcz, "Ribavirin Treatment in Dialysis Patients with Chronic Hepatitis C Virus Infection-A Pilot Study," Journal of Viral Hepatitis, Vol. 8, No. 4, 2001, pp. 287-292. doi:10.1046/j.1365-2893.2001.00300.x

[15] G. Mazzella, E. Accogli, S. Sottili, et al., "Alpha Interferon Treatment May Prevent Hepatocellular Carcinoma in HCV-Related Liver Cirrhosis," Journal of Hepatology, Vol. 24, No. 2, 1996, pp. 141-147. doi:10.1016/S0168-8278(96)80022-5

[16] M. Kohara, T. Tanaka, K. Tsukiyama-Kohara, et al., "Hepatitis C Virus Genotypes 1 And 2 Respond to Interferon-Alpha with Different Virologic Kinetics," Journal of Infectious Diseases, Vol. 172, 1995, pp. 934-938. doi:10.1093/infdis/172.4.934

[17] H. Nakamura, H. Ogawa, T. Kuroda, et al., "Interferon Treatment for Patients with Chronic Hepatitis C Infected with High Viral Load of Genotype 2 Virus," Hepatogastroenterology, Vol. 49, No. 47, 2002, pp. 1373-1376.

[18] J. Bukh, X. Forns, S. U. Emerson and R. H. Purcell, "Studies of Hepatitis C Virus in Chimpanzees and Their Importance for Vaccine Development," Intervirology, Vol. 44, No. 2-3, 2001, pp. 132-142.

doi:10.1159/000050040

[19] D. G. Bowen and C. M. Walker, "Mutational Escape from CD8+ T cell Immunity: HCV Evolution, from Chimpanzees to Man," The Journal of Experimental Medicine, Vol. 201, No. 11, 2005, pp. 1709-1714. doi:10.1084/jem.20050808

[20] M. Lechmann and T. J. Liang, "Vaccine Development for Hepatitis C," Seminars in Liver Disease, Vol. 20, No. 2, 2000, pp. 211-226. doi:10.1055/s-2000-9947

[21] S. Okada, H. Harada, T. Ito, T. Saito and S. Suzu, "Early Development of Human Hematopoietic and Acquired Immune Systems in New Born NOD/Scid/Jak3null Mice Intrahepatic Engrafted with Cord Blood-Derived CD34 + Cells," International Journal of Hematology, Vol. 88, No. 5, 2008, pp. 476-482. doi:10.1007/s12185-008-0215-Z

[22] S. Hattori, K. Ide, H. Nakata, et al., "Potent Activity of a Nucleoside Reverse Transcriptase Inhibitor, 4'-Ethynyl2-Fluoro-2'-Deoxyadenosine, against Human Immunodeficiency Virus Type 1 Infection in a Model Using Human Peripheral Blood Mononuclear Cell-Transplanted NOD/ SCID Janus Kinase 3 Knockout Mice," Antimicrob Agents
Chemother, Vol. 53, No. 9, 2009, pp. 3887-3893. doi:10.1128/AAC.00270-09

[23] F. Kobune, H. Sakata and A. Sugiura, "Marmoset Lymphoblastoid Cells as a Sensitive Host for Isolation of Measles Virus," Journal of Virology, Vol. 64, 1990, pp. 700-705.

[24] K. Tsukiyama-Kohara, S. Tone, I. Maruyama, et al., "Activation of the CKI-CDK-Rb-E2F Pathway in Full Genome Hepatitis C Virus-Expressing Cells," The Journal of Biological Chemistry, Vol. 279, No. 15, 2004, pp. 14531-14541. doi:10.1074/jbc.M312822200

[25] M. Satoh, M. Saito, K. Tanaka, et al., "Evaluation of a Recombinant Measles Virus Expressing Hepatitis C Virus Envelope Proteins by Infection of Human PBL-NOD/Scid/ Jak3null Mouse," Comparative Immunology, Microbiology and Infectious Diseases, Vol. 33, No. 6, 2010,pp. e81-e88. doi:10.1016/j.cimid.2010.02.006

[26] M. Yoneda, R. Miura, T. Barrett, K. Tsukiyama-Kohara and C. Kai, "Rinderpest Virus Phosphoprotein Gene is a Major Determinant of Species-Specific Pathogenicity," Journal of Virology, Vol. 78, No. 12, 2004, pp. 6676-6681. doi:10.1128/JVI.78.12.6676-6681.2004

[27] A. Op De Beeck, L. Cocquerel and J. Dubuisson, "Biogenesis of Hepatitis C Virus Envelope Glycoproteins," Journal of General Virology, Vol. 82, No. 11, 2001, pp. 2589-2595.

[28] E. Falkowska, F. Kajumo, E. Garcia, J. Reinus and T. Dragic, "Hepatitis C Virus Envelope Glycoprotein E2 Glycans Modulate Entry, CD81 Binding, and Neutralization," Journal of Virology, Vol. 81, No. 15, 2007, pp. 8072-8079. doi:10.1128/JVI.00459-07

[29] F. Helle, A. Goffard, V. Morel, et al., "The Neutralizing Activity of Anti-hepatitis C Virus Antibodies is Modulated by Specific Glycans on the E2 Envelope Protein," Journal of Virology, Vol. 81, No. 15, 2007, pp. 8101-8111. doi:10.1128/JVI.00127-07

[30] P. Jackson, J. Petrik, G. J. Alexander, G. Pearson and J. P. Allain, "Reactivity of Synthetic Peptides Representing Selected Sections of Hepatitis C Virus Core and Envelope Proteins with a Panel of Hepatitis C Virus-Seropositive Human Plasma," Journal of Medical Virology, Vol. 51, No. 1, 1997, pp. 67-79.

doi:10.1002/(SICI)1096-9071(199701)51:1<67::AID-JM V11>3.0.CO;2-1

[31] J. H. Hoofnagle, "Course and Outcome of Hepatitis C," Hepatology, Vol. 36, No. 5, 2002, pp. S21-S29. doi:10.1002/hep.1840360704 